\title{
Alter
}

Revue de phénoménologie

19 | 2011

Le langage

\section{L’usage phénoménologique du langage indexical}

\section{Vincent Grondin}

\section{OpenEdition}

\section{Journals}

Édition électronique

URL : http://journals.openedition.org/alter/1392

DOI : $10.4000 /$ alter.1392

ISSN : 2558-7927

\section{Éditeur :}

Association ALTER, Archives Husserl (CNRS-UMR 8547)

\section{Édition imprimée}

Date de publication : 1 octobre 2011

Pagination : 123-140

ISBN : 978-2-9522374-7-5

ISSN : 1249-8947

Référence électronique

Vincent Grondin, «L'usage phénoménologique du langage indexical », Alter [En ligne], 19 | 2011, mis en ligne le 01 janvier 2020, consulté le 23 janvier 2020. URL : http://journals.openedition.org/alter/1392 : DOI : 10.4000/alter.1392 


\title{
L’USAGE PHÉNOMÉNOLOGIQUE DU LANGAGE INDEXICAL
}

\author{
Vincent Grondin
}

Selon une représentation pratique et commode que l'on se fait généralement de la phénoménologie husserlienne, cette dernière se caractériserait par la thèse selon laquelle le langage n'épuise pas le régime du sens ${ }^{1}$. En effet, bien qu'il protestait à l'epoque des Recherches logiques contre le caractère " artificiel » de la distinction frégéenne du Sinn et de la Bedeutung², Husserl n'hésitera pas dès le premier tome des Idées directrices à employer la notion de Sinn pour désigner le « sens » pré-linguistique qui, par opposition à la Bedeutung linguistique, organise et structure la perception de tout objet ${ }^{3}$. Comme chacun sait, cet élargissement de la notion de sens culminera dans l'affirmation célèbre des Méditations cartésiennes selon laquelle la tâche de la phénoménologie est de porter à l'expression le «sens»(Sinn) de $l^{\prime}$ « expérience encore muette $»^{4}$.

La thèse husserlienne selon laquelle le langage se contenterait de donner une forme expressive à un sens qui se manifeste au sein même de l'expérience a fait l'objet de critiques vives et énergiques. S'il faut en croire des auteurs aussi différents que Philipp Pettit et

\footnotetext{
${ }^{1}$. C'est du moins la définition célèbre qu'en propose Merleau-Ponty dans la Préface de la Phénoménologie de la perception. M. Merleau-Ponty, Phénoménologie de la perception, Paris, Gallimard, 1945, p. X.

2. E. Husserl, Logische Untersuchungen, Untersuchungen zur Phänomenologie und Theorie der Erkenntnis, $2^{\mathrm{e}}$ tome, $1^{\text {re }}$ partie, Tübingen, Max Niemeyer Verlag, p. 53; tr. fr. H. Elie, A. L. Kelkel et R. Schérer, Recherches logiques. Recherches pour la phénoménologie et la théorie de la connaissance, Paris, Presses universitaires de France, 1961, p. 62 (désormais cité: RL II/1, suivi de la pagination allemande puis, entre crochets, de la pagination de traduction française).

${ }^{3}$. E. Husserl, Ideen zu einer reinen Phänomenologie und Phänomenologie und phänomenologischen Philosophie I, Max Niemeyer Verlag, Tübingen, 1993, § 124, p. 256; tr. fr. P. Ricœur, Idées directrices pour une phénoménologie et une philosophie phénoménologique pures I. Introduction générale à la phénoménologie pure, Paris, Gallimard, § 124, p. 419 (désormais cité : Ideen I).

${ }^{4}$. E. Husserl, Cartesianische Meditationen und Pariser Vorträge, éd. S. Strasser, Husserliana I, $2^{\mathrm{e}}$ édition, Den Hague, Martinus Nijhoff, 1970, p. 77; tr. fr. M. de Launay, Méditation cartésiennes et Les conferences de Paris, Paris, Presses universitaire de France, 1994, § 16, p. 84 (désormais cité : $C M)$.
} 
Jacques Derrida, la phénoménologie découle d'une conception angélique du langage qui a conduit Husserl à sous-estimer le fait que nos vécus psychiques sont toujours "médiatisés » et "altérés » par un « filtre » langagier 5 . Sans nul doute, un tel reproche fait montre d'une certaine mauvaise foi qui mène les contempteurs de la phénoménologie à négliger certains faits textuels troublants qui montrent bien que les réflexions de Husserl sur le langage et la nature de son propre discours s'avèrent beaucoup plus complexes que l'on a pu le croire à une certaine époque. Par exemple, on ne peut que s'étonner de voir Husserl faire appel, dans les Idées directrices notamment, à un dispositif éminemment linguistique pour expliquer et thématiser la teneur de la méthode phénoménologique. Selon une formule étrange du $\$ 89$ des Idées directrices, la description phénoménologique porte sur l'objet entre guillemets 6 . Dans ce passage, Husserl trace alors une distinction entre l'objet physique et effectif et l'objet tel qu'il est perçu par la conscience. Après avoir mis en place cette distinction importante, Husserl souligne que seul est pertinent pour la phénoménologie " l'objet entre guillemets », c'est-à-dire l'objet tel qu'il est perçu.

Sans surprise, cet usage des guillemets est a l'origine de deux réactions diamétralement opposées. Alors que des auteurs tels Smith et McIntyre en tirent la conclusion que l'on peut interpréter toutes les descriptions phénoménologiques comme l'analyse logique d'énoncés perceptuels guillemetés, Derrida soutient au contraire que cet usage étrange des guillemets atteste que la distinction des vécus préexpressifs et de l'expression linguistique est équivoque et trahit certains présupposés métaphysiques ${ }^{7}$. Dans un cas comme dans l'autre, on ne peut s'empêcher de déplorer que des jugements philosophiques aussi importants reposent finalement sur un examen plutôt sommaire et expéditif des textes où Husserl prend le soin de préciser de quelle manière l'analogie des guillemets permet de définir la nature de l'attitude phénoménologique. Dans ce qui suit, je me propose d'expliquer d'une manière détaillée l'usage méthodologique que fait Husserl des guillemets en prenant pour échantillon l'emploi

\footnotetext{
5. Cf. P. Pettit, "On phenomenology as a methodology of Philosophy », in Linguistic Analysis and Phenomenology, Glasgow, Macmillan Press, 1972, p. 246 et J. Derrida, «La forme et le vouloirdire : note sur la phénoménologie du langage ", in Marges de la philosophie, Paris, Éditions de Minuit, 1972, p. 187-207.

${ }^{6}$. Ideen I, § 89, p. 184, [p. 308].

7. Smith et McIntyre interprètent les descriptions noématiques comme des "citations noématiques » qui, à l'image des énoncés d'attitude propositionnelle, peuvent faire l'objet d'une analyse logique de type intensionnelle. Cf. D. Smith et R. McIntyre, Husserl and Intentionality: A study of Mind, Meaning and Language, Boston, D. Reidel Publishing Company, 1982, p. 190 (toutes mes références à Smith et McIntyre renvoient à cet ouvrage). Loin de voir dans cet usage une innovation géniale, Derrida soutient qu'elle ne fait que reconduire la tension qu'il y a entre l'usage que nous faisons du langage dans l'attitude naturelle et le sens que le phénoménologue donne au concept d'objet. Cf. J. Derrida, La voix et le phénomène, Paris, Presses universitaires de France, 1967, p. 58.
} 
qu'il en fait dans le cadre de ses réflexions sur l'organisation « indexicale » de l'expérience vécue (§ 2-3). Toutefois, avant d'aborder cette question délicate et épineuse, j'aimerais faire quelques remarques d'ordre général qui me permettront de préciser la signification que possèdent les guillemets pour Husserl (§ 1).

\section{Guillemets et attitude phénoménologique}

Dans la mesure où Husserl n'a jamais expliqué d'une manière précise et exhaustive la fonction que jouent les guillemets dans l'économie de son discours, le travail de reconstruction s'avère particulièrement complexe et délicat. Il est en effet inévitable de faire appel à des passages tirés de textes appartenant à des périodes différentes afin de montrer la profonde cohérence des vues de Husserl relativement à cette question.

En dépit de cette difficulte, il est possible d'énoncer deux grandes thèses qui résument $d$ 'une manière claire et nette la conception husserlienne des guillemets : 1/ Les guillemets permettent de souligner l'originalité de la phénoménologie par rapport à l'ontologie et la psychologie. 2/ Les guillemets témoignent du type d'objectivation qui est au fondement du discours phénoménologique. - Afin d'aller directement à l'essentiel, je vais me contenter d'expliquer rapidement ces deux affirmations qui jouent un rôle cardinal dans la définition de la phénoménologie.

\section{a) La phénoménologie comme étude de l'« objet » et du «vécu »}

Dans un passage déterminant $\mathrm{du}$ troisième volume des Idées directrices, Husserl définit le registre de son discours en mentionnant que la phénoménologie, au contraire de l'ontologie, porte sur l'objet entre guillemets :

En la qualité de phénoménologues, nous effectuons aussi des positions, des prises de position actuelle, mais elles sont exclusivement dirigées sur les vécus et sur les corrélats des vécus. En ontologie, à l'encontre, nous effectuons des positions actuelles qui, au lieu d'être dirigées sur les corrélats et sur les objets entre guillemets, le sont sur les objets purs et simples ${ }^{8}$.

\footnotetext{
${ }^{8}$. E. Husserl, Ideen zu einer reinen Phänomenologie und phänomenologischen Philosophie III. Die Phänomenologie und die Fundamente der Wissenschaften, éd. M. Biemel, Husserliana V, (Den Hague, Martinus Nijhoff, 1971), p. 88; tr. fr. A. L. Kelkel, Idées directrices pour une phénoménologie et une philosophie phénoménologiques pures III. La phénoménologie et les fondements des sciences, Paris, Presses universitaires de France, 1993, p. 105 (désormais cité : Ideen III).
} 
Tandis que le domaine de l'ontologie est l'étant, c'est-à-dire les structures formelles et matérielles de l'objectivité, la phénoménologie est la science des "évènements transcendantaux », c'est-à-dire des actes par lesquels tout objet se constitue pour la conscience ${ }^{9}$. Ceci ne veut bien sûr pas dire que l'objectivité n'a aucune pertinence phénoménologique et qu'elle doive être tout simplement laissée de côté. Puisque les actes intentionnels de la conscience possèdent systématiquement un corrélat objectif, la question du statut de l'objectivité concerne la phénoménologie. Certes, les modalités d'existence de l'objectivité ne relèvent pas du cahier des charges de la phénoménologie. Il n'en demeure pas moins qu'elle doit décrire comment la conscience se rapporte à l'objectivité. En d'autres termes, l'objet relève de l'enquête de la phénoménologie dans l'exacte mesure où on l'appréhende comme un noème, c'est-a-dire comme le corrélat d'un acte de visée.

L'intentionnalité se définissant comme la visée d'un objet, l'acte intentionnel véhicule une certaine prétention à l'égard de l'objectivité. La perception est la perception de tel ou tel objet, le jugement est le jugement de tel ou tel état de choses, etc. Dès lors, le quadrillage du champ phénoménologique s'appuie sur la distinction de l'objet effectif et de l'objet présumé, c'est-à-dire l'objet tel qu'il est visé par un certain acte :

Décrire et déterminer conformément à l'essence le phénomène d'intuition de chose [...], cela veut dire en même temps tenir compte du fait que l'acte est en lui-même un "présumer » quelque chose et que le présumé, en tant que tel, est une "chose». Or, constater cela et faire de la chose présumée en tant que telle [...] l'objet de la recherche, former des énoncés sur ce qui revient eidétiquement à ces sortes de choses présumées en tant que telles, ne signifie pas examiner les choses, les choses comme telles ${ }^{10}$.

En somme, ce qui doit retenir l'attention du phénoménologue n'est pas de savoir comment les choses sont, mais comment elles sont visées et posées par les actes intentionnels. L'usage des guillemets s'avère donc capital, car il ne vient marquer rien d'autre que cet écart. Comme le note Husserl dans une formule lapidaire : "Une "chose" comme corrélat n'est pas une chose : d'où les guillemets ${ }^{11}$. La phénoménologie ne porte pas sur les choses, mais bien sur les " choses ", c'est-à-dire les corrélats présumés des actes intentionnels.

Il est à noter que les guillemets n'ont pas pour seule vocation de creuser l'écart entre deux manières de se rapporter à l'objectivité. Dans le passage cité un peu plus haut, «chose »n'est pas le seul

\footnotetext{
9. Ideen III, p. 84, [p. 100].

${ }^{10}$. Ideen III, p. 84, [p. 101].

11. Ibid.
} 
terme à avoir été encadré de guillemets. Le mot "présumer » qui vient qualifier l'activité de la conscience est aussi accompagné de guillemets. Si les guillemets sont indispensables pour dissocier la phénoménologie de l'ontologie dans leur traitement de l'objectivité, ils permettent aussi de distinguer la phénoménologie de la psychologie. A l'instar de l'ontologie qui porte sur les objets en tant qu'ils existent, la psychologie s'intéresse aux vécus psychiques en tant qu'ils sont des faits empiriques. Lorsqu'on adopte l'attitude de la psychologie, on considère la subjectivité comme une âme, c'est-à-dire qu' on la conçoit comme un objet, comme un morceau du monde nature. Une telle approche est tout à fait étrangère à la perspective phénoménologique qui considère au contraire la subjectivité en tant que pure subjectivite ${ }^{12}$. Dans un tel contexte, le regard phénoménologique ne porte pas sur la conscience en tant que chose naturelle, mais sur la conscience en tant que conscience. Pour reprendre la formulation de la Postface à mes Idées directrices : " Notre regard libéré par cette épochè s'ouvre alors sur le phénomène universel: "1'univers de la conscience comme tel", purement comme celui qui nous est connu à travers l'écoulement de la conscience $»^{13}$.

Afin de mettre en relief ce «comme tel » et cet « en tant que » qui sont constitutifs de l'attitude phénoménologique à l'égard des vécus, Husserl n'hésite pas à faire appel aux guillemets. Dans un passage clé de la Postface à mes Idées directrices qui souligne comme bien d'autres que la réflexion phénoménologique porte sur le « je transcendantal » et non pas la personne humaine que je suis, Husserl fait la remarque suivante: "Continuant ainsi ma médiation, je comprends également à présent que mon essence propre phénoménologiquement close peut être posée d'une manière absolue, comme "je", ce "je" que je suis »14. Considérant que la phénoménologie possède une double orientation, à savoir subjective et objective, on pourrait dire que les guillemets représentent de part et d'autre un dispositif pour marquer le territoire de la phénoménologie. Tandis que l'ontologie porte sur la chose, la phénoménologie étudie la "chose ». De même, à la différence de la psychologie qui étudie l'âme, c'est-à-dire le je réel en tant qu'il renvoie à une personne humaine, la phénoménologie a pour objet d'étude la subjectivité en tant que subjectivité, c'est-à-dire le «je » que je suis.

\footnotetext{
12. Ceci est très clair lorsqu'on considère la description de l'épochè proposée par la Postface à mes Idées directrices : "La nature organique et psychique, le monde en général comme totalité qui constitue pour moi dans l'attitude naïve l'étant pur et simple, perd par l'épochè phénoèménologique sa valeur d'être ». (Ideen III, p. 145, [p. 188]). En d'autres termes, ce qui fait du sujet un étant, une chose qui appartient au monde de la nature est mis hors circuit.

13. Ideen III, p. 145, [p. 189].

14. Ideen III, p. 149, [p. 194].
} 


\section{b) La «transformation réifiante »}

Rendu à ce stade, on doit cependant s'empresser de souligner avec force que cet usage des guillemets n'a pas pour simple fonction de marquer un simple changement $d^{\prime}$ attitude. Les guillemets s'imposent aussi dans le présent contexte parce que la phénoménologie prend pour « objet » d'étude des phénomènes (comme les vécus psychiques, les structures générales de l'intentionnalité, les formes catégoriales de l'objectivité et les significations) qui, justement, ne sont pas, au sens strict du terme, des objets. Si Amie Thomasson a raison de soutenir que la réduction phénoménologique implique une "réification » qui transforme les phénomènes en " objets », ceci signifie que Husserl devait nécessairement avoir recours au dispositif typographique des guillemets pour thématiser le registre de son propre discours ${ }^{15}$. Il $\mathrm{s}^{\prime}$ agit en effet d'une thèse explicitement soutenue par Husserl à l'époque des Leçons sur la théorie de la signification de 1908 : l'objectivation des phénomènes qui, selon Husserl, est rendue possible grâce à un « acte nominatif », peut être désignée à l'écrit par les guillemets.

Selon la théorie avancée par les Leçons, le jugement s'organise essentiellement autour de deux pôles qui correspondent à deux attitudes différentes que l'on peut adopter à l'égard de l'objet. D'une part, l'objet apparaît à la conscience sous une certaine forme catégoriale. Par exemple, lorsque je songe à Napoléon, je ne songe pas seulement à un personnage historique, mais je pense à lui selon une certaine guise. Si je pense au perdant de Waterloo ou au vainqueur $\mathrm{d}^{\prime}$ Iéna, la représentation que je $\mathrm{m}^{\prime}$ en fais est différente ${ }^{16}$. Cette différence s'explique par le fait que ma visée de Napoléon n'est pas la même d'un point de vue catégorial. Vaincre à Iéna n'équivaut pas à perdre à Waterloo. Pour employer le jargon des Leçons, disons que les actes prédicatifs qui relient le jugement à son objet sont différents ${ }^{17}$. Ceci ne veut pas dire - Husserl est très clair à ce propos - que les actes prédicatifs ne se rapportent pas à un objet cohérent et unitaire. Seulement, lorsqu'on se situe à l'échelon des actes prédicatifs, l'objet correspond à l'intersection des différents actes prédicatifs.

\footnotetext{
15. A. L. Thomasson, "First-Person Knowledge in Phenomenology», in Phenomenology and Philosophy of Mind, Oxford et New York, Oxford University Press, 2005, p. 131-132.

16. E. Husserl, Vorlesungen über Bedeutungslehre. Sommersemester 1908, éd. U. Panzer, Husserliana XVI, (Dordrecht, Boston, Lancaster, Martinus Nijhoff, 1987), p. 36; tr. fr. J. English, Paris, Vrin, 1987, p. 60 (désormais cité : Beudeutungslehre).

17. Husserl propose au $\S 17$ la définition suivante des actes prédicatifs ; « Les actes qui confèrent la signification sont des actes prédicatifs, c'est-à-dire des actes qui ou bien sont des prédications pleines ou peuvent s'insérer, en tant que parties constitutives immédiates propres, dans des prédications. Et c'est seulement dans leur fonction prédicative qu'ils montrent leur relation objective ». Bedeutungslehre, p. 64, [p. 89]. Les actes prédicatifs sont donc des actes qui visent un objet en le déterminant, en lui attribuant des prédicats.
} 
L'acte prédicatif n'épuise cependant pas la complexité du concept d'objet. Il est possible de court-circuiter les déterminations prédicatives de l'objet et de viser le substrat de toutes ces déterminations. En d'autres termes, tous ces prédicats doivent être supportés par un substrat qui peut être visé par un acte dit «nominal » :

\begin{abstract}
À l'intérieur de la prédication, les actes nominaux ont une position insigne. Ils sont représentatifs en un sens prégnant. Ils constituent dans la conscience prédicative les objets-sur-quoi; surtout, ils font cela, au sens le plus étroit, en tant qu'actes de sujet dans lesquels les objets se tiennent en tant que tels, "sur lesquels porte l'énoncé » ${ }^{18}$.
\end{abstract}

Comme l'annonce ce passage, le support objectif de la prédication est, selon la terminologie de Husserl, un « objet-sur-quoi » (Gegenstand-worüber), qui est posé par un acte nominal. L'acte nominal est l'équivalent logique de l'emploi d'un nom propre permettant de viser l'objet auquel se rapportent les différents prédicats.

Les guillemets interviennent dans l'analyse de Husserl lorsqu'il tente d'appliquer ce modèle au registre même de la signification. Chaque acte prédicatif, en tant qu'intention de signification, vise l'objet d'une certaine manière. Mais que se passe-t-il lorsqu'on tente de poser un jugement sur une signification ? Dans ce cas de figure, la forme catégoriale ne fait plus partie de la visée qui nous donne accès à l'objet, mais elle devient l'objet même sur lequel porte notre jugement. Autrement dit, par un acte nominal, la signification devient un « objet-sur-quoi ». Et c'est dans ce cadre que les guillemets entrent en scène :

Là aussi se séparent l'attitude nominale non réfléchie dans laquelle l'objetsur-quoi est précisément objet, et celle, catégorialement réfléchie, dans laquelle le catégorial est objectif. Et ceci aussi ne peut pas être indiqué autrement que par la répétition de l'expression nominale complexe entière: mais, dans le mode d'expression écrit, nous employons des guillemets ${ }^{19}$.

Notre rapport à l'objet est médiatisé par une forme catégoriale, mais cette forme elle-même n'est pas (toujours) l'objet de notre attention. Ceci ne nous empêche pas d'élever cette catégorialité à la réflexion et d'en faire l'objet d'une visée. Dans ce contexte, les guillemets représentent le dispositif typographique qui permet de désigner ce changement d'attitude.

On ne doit pas s'étonner que ces réflexions sur les guillemets soient enchâssées dans le cadre plus général d'une théorie de la signification. Déjà à l'époque des Recherches logiques, Husserl avait été frappé par le phénomene des guillemets qui permet $\mathrm{d}^{\prime}$ arracher une

18. Bedeutungslehre, p. 66, [p. 91].

19. Bedeutungslehre, p. 86, [p. 113]. 
expression à ses coordonnées grammaticales habituelles pour modifier le regard que nous rejetons sur sa signification. Toutefois, l'originalité de l'usage des guillemets qu'en fait la phénoménologie transcendantale réside dans l'élargissement de ce modèle. Non seulement les significations peuvent, grâce aux guillemets, devenir des « objets » de discours, mais il est possible de répéter la manœuvre pour caractériser le regard que le phénoménologue jette sur les vécus et les objets tels qu'ils se donnent dans la perception.

Pour résumer, en plus de permettre de départager la phénoménologie de l'ontologie et de la psychologie, les guillemets attestent aussi de l'originalité du discours phénoménologique qui consiste à prendre pour objet d'étude des structures aprioriques - la plupart du temps subjectives - qui ne sont justement pas des objets. Comme nous allons le voir, c'est précisément un tel geste qui est à l'œuvre dans l'usage fait par les Méditations cartésiennes des expressions indexicales. En encadrant de guillemets les expressions " je », « ici », " là-bas », Husserl nous rappelle que ses analyses ont pour objet certaines structures aprioriques qui donnent sa forme indexicale et égocentrique à l'expérience vécue que nous faisons tous du monde ambiant.

\section{Le langage indexical : formulation du problème}

Aron Gurwitsch a indéniablement raison lorsqu'il remarque qu'une description phénoménologique de notre expérience perceptuelle serait tout simplement impossible sans le recours à des expressions indexicales telles que «je», «ici » ou «maintenant $»^{20}$. Aussi évident que puisse être ce constat, il soulève tout de même une difficulté de taille puisque le $\S 25$ de la première Recherche proclame que l'idée même de scientificité exige $\mathrm{qu}^{\prime}$ il soit en droit possible d'imaginer un langage purgé de toute indexicalité.

Selon la conception de la science avancée par les Recherches logiques, les énoncés scientifiques doivent être universellement valables, c'est-à-dire qu'ils doivent pouvoir être répétés et réactivés à l'infini; ils ne peuvent donc pas varier en fonction du contexte. Un énoncé scientifique doit être un énoncé objectif, c'est-à-dire un énoncé dont la signification demeure toujours une et identique. Dans ce texte crucial, Husserl dresse une distinction entre les expressions subjectives dont la signification varie en fonction du contexte d'énonciation et les expressions objectives dont la signification reste toujours stable. Or, d'après Husserl, l'idée de science est intimement liée à un principe

20. G. Gurtwitsch, "Outlines of a Theory of "Essentially Occasional Expressions" ", Readings on Edmund Husserl's Logical Investigations, Den Hague, Martinus Nijhoff, 1977, p. 120-121. 
d'absence de limite de la raison objective qui stipule que les expressions subjectives n'appartiennent pas à l'essence du langage. En principe, l'idée d'un langage purement scientifique et objectif a un sens. Il est ainsi théoriquement possible d'imaginer un langage purifié de ses ambiguités et de la variabilité de la signification de ses expressions ${ }^{21}$.

Ce principe est inquiétant, car il semble autoriser, en droit du moins, l'élimination de la phénoménologie qui repose sur un certain usage des indexicaux ayant pour fonction d'exhiber la structure egocentrique de l'expérience subjective qui se distribue en fonction de $l^{\prime}$ « ici » et du «maintenant ». En prenant une perspective légèrement différente, on pourrait d'ailleurs aussi se demander si l'idée même d'une "science » de la subjectivité peut avoir un sens. Bien que la phénoménologie réclame le titre de "science », le fait demeure que la phénoménologie, en tant que science de la subjectivité, se caractérise par ce que Husserl nomme au $\S 13$ des Méditations cartésiennes une «singularité inouïe ». Le passage est long, mais on doit le citer dans son intégralité :

\begin{abstract}
Une science d'une singularité inoüe se dessine à nos yeux, une science de la subjectivité transcendantale concrète, donnée dans l'expérience transcendantale possible et réelle, qui représente l'opposé le plus extrême des sciences au sens qu'on leur a donné jusqu'ici, aux sciences objectives. Parmi celles-ci, il y a certes aussi une science de la subjectivité, mais il s'agit de la subjectivité objective, animale, qui ressortit au monde. Maintenant, au contraire, il s'agit d'une science pour ainsi dire absolument subjective, d'une science dont l'objet est, dans son être, indépendant de la décision sur le non-être ou l'être du monde ${ }^{22}$.
\end{abstract}

Ce texte fascinant soulève une difficulté redoutable. On pourrait effectivement se demander si la "singularité inouïe » de la phénoménologie ne revient pas à remettre en question son statut de science. Pour rebondir sur la distinction tracée par les Recherches logiques entre les expressions subjectives et les expressions objectives, on pourrait en effet se demander s'il est possible de développer une science, même une science de la subjectivité, en faisant appel à des énoncés «subjectifs» et indexicaux. L'usage des indexicaux ne vient-il pas remettre en cause la prétention à l'universalité de la science phénoménologique?

Afin de dissoudre cette tension, il est important de souligner que Husserl attribue deux significations profondément différentes au concept d'objectivité. Si le § 13 des Méditations cartésiennes soutient bel et bien que la phénoménologie n'est pas une science "objective", $c^{\prime}$ est au sens où la phénoménologie ne porte pas, à la différence des

${ }^{21}$. RL II/1, p. 90, [p. 105].

22. CM, p. 68, [p. 74]. 
sciences empiriques, sur des « objets mondains ». Toutefois, cela ne veut pas dire que le discours phénoménologique n'est pas " objectif » en un autre sens. Si certains énoncés sont dits " objectifs" au sens où les Recherches logiques emploient se terme, ce n'est pas parce qu'elles portent sur des objets, mais c'est bien plutôt parce que leur signification possède une identité idéale qui leur confère une certaine universalité et, par conséquent, une forme d'objectivité. Comme le note Husserl au § 29 de la première Recherche, la science n'est pas un amalgame subjectif et contingent de représentations mentales. La science est au contraire une complexion de significations reliées par des rapports idéaux et nécessaires qui ne sont pas le produit d'un sujet individuel et, par conséquent, lesdits rapports possèdent une certaine objectivitéz 3 . À partir du moment ou la phénoménologie entreprend d'exhiber les connexions idéales et nécessaires qu'il y a entre les différents vécus et leurs éléments, rien n'empêche la phénoménologie d'être une science " objective » de la subjectivité.

On l'aura compris, le tour de force de la phénoménologie consiste justement à construire un discours " objectif » à portée universelle qui repose pourtant sur un certain usage du langage indexical. La fin du $\S 13$ de Chose et espace ne laisse place à aucun doute à cet égard :

Le droit que l'on a de rapporter les formations de conscience à un Je, à cette personne-ci ou à cette personne-là, doit être d'abord fondé par la pensée objectivante et sa logique; et ce droit légitime son sens dans l'analyse phénoménologique. Mais la pensée dont elle parle n'est la pensée de personne ${ }^{24}$.

Les structures intentionnelles n'appartiennent à aucun sujet en particulier. Elles sont en réalité des structures anonymes qui représentent les conditions de possibilité de toute expérience - peu importe ici que cette expérience soit faite par moi ou par un autre. Lorsque je me livre à l'activité phénoménologique, mon objectif n'est pas de décrire dans leur actualité et leur singularité les vécus qui composent ma vie intentionnelle. Non, l'objectif de la phénoménologie est de décrire ma vie intentionnelle dans ce qu'elle a d'anonyme. Par exemple, lorsque le phénoménologue étudie la perception, il ne s'intéresse pas à ce que telle ou telle perception a de singulier. $\mathrm{Au}$ contraire, il s'agit de déterminer les propriétés essentielles qui caractérisent tout vécu de perception. Et c'est uniquement dans la mesure où la phénoménologie décrit effectivement de telles structures universelles qu'elle échappe à l'objection du subjectivisme et

\footnotetext{
${ }^{23}$. RL II/1, p. 95, [p. 108].

24. E. Husserl, Ding und Raum. Vorlesungen 1907, éd. U. Claesges, Husserliana XVI, (Den Hague, Martinus Nijhoff, 1973), p. 41; tr. fr. J.-F. Lavigne, Chose et espace, Paris, Presses universitaires de France, 1987, p. 64.
} 
qu'elle a effectivement raison de prétendre que les énoncés qu'elle produit possèdent bel et bien une certaine objectivité.

Bien évidemment, Husserl n'exclut pas que le phénoménologue se livre, ponctuellement, à une description de sa propre expérience. En tant que phénoménologue, je peux très bien faire la description de $l^{\prime}$ ego transcendantal singulier que je suis ${ }^{25}$. Une telle entreprise d'explicitation de mon expérience possède néanmoins une certaine naïveté empirique qui, d'un point de vue phénoménologique, doit être dépassée. Ce qui intéresse la phénoménologie en dernière instance, ce n'est pas mon expérience en tant que sujet individuel particulier. Pour le dire dans les termes de Husserl, le phénoménologue focalise son attention sur la forme universelle de l'ego, sur l'eidos de l'ego. Même si le phénoménologue n'utilise pas toujours en un sens phénoménologique le pronom «je ", l'usage proprement phénoménologique vise une structure universelle. Comme le note Husserl : « La phénoménologie eidétique cherche donc l'a priori universel sans lequel ne sont pas concevables ni le je ni un moi transcendantal en général $\aleph^{26}$. Dès lors, quoique le phénoménologue continue à utiliser toute une série d'expressions indexicales, il leur injecte une signification objective en visant, par ses analyses, les structures aprioriques et universelles de la subjectivité transcendantale en général.

Une fois cette clarification faite, on pourrait se demander ce que l'usage phénoménologique des expressions indexicales conserve de leur indexicalité. Au §34 des Méditations cartésiennes, Husserl soutient que les structures universelles et eidétiques de la subjectivité ne sont conditionnées par aucun fait et, par conséquent, elles ne sont pas le produit de l'évolution contingente de notre langage :

L'eidos lui-même est un universel vu ou visible, un universel pur, inconditionné, c'est-à-dire conditionné par aucun fait, conformément à son propre sens intuitif. Il précède tous les concepts au sens de significations verbales (Wortbedeutungen), lesquelles, comme purs concepts, doivent au contraire être formés en s'adaptant à lui $i^{27}$.

La phénoménologie a pour projet de décrire les structures fondamentales de la subjectivite qui ont un «sens intuitif » et qui se situent en amont des significations verbales et langagières (Wortbedeutungen). Se pose donc dans toute son ampleur la question du rapport que la grammaire de l'indexicalité entretient avec les structures eidétiques qui font l'objet de l'enquête phénoménologique. Poser cette question, $c^{\prime}$ est par le fait même soulever le problème de la nature de l'usage fait par la phénoménologie des expressions indexicales, usage qui per-

\footnotetext{
25. CM, p. 103, [p. 117].

26. CM, p. 105, [p. 119].

27. CM, p. 105, [p. 118].
} 
met, du moins en apparence, de court-circuiter leur signification verbale.

\section{La signification « anormale » des expressions indexicales employée par la phénoménologie}

Que le discours phénoménologique repose sur un usage étonnant et inusité des expressions indexicales, voilà qui est évident. Pourtant, cette modification n'est pas si extraordinaire qu'on pourrait le croire au premier abord. En fait, l'usage "phénoménologique » des indexicaux ne repose sur rien d'autre que la distinction des significations normales et anormales évoquée par Husserl dans la théorie des expressions indexicales avancée par le troisième chapitre de la premiere Recherche logique. Dans ce passage, Husserl aborde la question de ce qu'il nomme lui-même les "expressions occasionnelles ». Ce terme technique enveloppe toutes les expressions qui varient en fonction de leur contexte d'énonciation. Les expressions indexicales ( « moi », « toi », « ici », « maintenant», etc.) représentent sans doute l'exemple le plus évocateur de cette variation de la signification. Lorsque le même mot «je » est employé par Olivier ou Maude, la personne désignée n'est pas la même et, par conséquent, la signification est différente ${ }^{28}$. Une telle variation de la signification n'est pourtant pas problématique. Dans la plupart des cas, c'est le contexte qui nous permet de déterminer la référence d'une expression indexicale. Par exemple, si j'emploie le mot «je»pendant une conférence, l'auditoire sait immédiatement de qui je parle.

Lorsque je comprends ce qu'une expression indexicale désigne, je saisis la signification «normale » de cette expression. Par contre, si je lis une note laissée sur la table dont je n'arrive pas à déterminer $l^{\prime}$ auteur, le mot «je » n'a pas sa signification normale ${ }^{29}$. Une profonde vérité est épinglée ici : même lorsque je ne suis pas en mesure de déterminer la référence d'une expression indexicale, il n'en demeure pas moins que cette expression a tout de même une signification minimale.

Afin de faire droit à cette possibilité, Husserl propose de distinguer deux étages de signification dans le cas des expressions indexicales. D'une part, l'expression indexicale possède une signification générale et universelle qui fait en sorte que, même dans le cas où le référent est indéterminé, le mot « je » a tout de même un sens qui fait en sorte que l'énoncé " je reviens dans cinq minutes » a un sens (une signification indicative). Néanmoins, les indexicaux ont aussi une 
signification qui, habituellement, permet de spécifier la référence de l'expression. Lorsque je dis « je », les mots que je prononce renvoient à une et une seule personne (une signification indiquée) ${ }^{30}$. Il est ici très tentant d'expliquer l'articulation de ces deux modalités de signification en ayant recours à la notion de contexte. L'analyse des indexicaux proposée par les Recherches logiques préfère cependant faire appel au vocabulaire de l'intuition. Normalement, l'expérience perceptuelle qui accompagne l'usage des indexicaux me permet d'identifier leur référence. Lorsque ce n'est pas le cas, ces expressions conservent tout de même une signification qui découle du rapport de dépendance qui caractérise normalement la signification des indexicaux et l'expérience intuitive ${ }^{31}$. Lorsque mon interlocuteur me dit «Regarde ceci! », je suppose automatiquement qu'il désigne un certain objet se manifestant au sein de son expérience intuitive.

En somme, l'expression indexicale dite normale possède deux niveaux de signification (générale et individuante). Et, inversement, l'expression indexicale a un défaut de signification, elle est dite " anormale », lorsqu'elle ne parvient pas à identifier l'objet particulier qu'elle désigne. Dans le jargon de Husserl, le déictique " anormal » est donc celui qui ne parvient qu'à exprimer sa signification universelle.

Puisque la phénoménologie fait un usage strictement "objectif » des indexicaux, seule la signification " anormale » et "générale » de ces expressions s'avère pour elle pertinente. Dès lors, le problème de l'écriture phénoménologique devient le suivant: quel dispositif grammatical pouvons-nous employer pour purger l'expression indexicale de sa signification normale qui la rattache à un contexte ponctuel et individuel? Dans les Recherches logiques, Husserl ne répond pas explicitement à cette question pour la simple raison qu'il suffit de passer à l'expression écrite pour que les indexicaux, dans certains contextes, perdent leur signification normale. Lorsque je lis une note affirmant: "Je serai de retour dans cinq minutes", je dois en connaître l'auteur pour que le mot « je » ait sa signification " normale». Bref, c'est le contexte qui permet de déterminer si une expression indexicale a bel et bien sa signification normale.

Pourtant, ce cas de figure n'est pas le seul envisagé par Husserl pour rendre compte du dualisme des significations normales et anormales. Au sein de la quatrième Recherche, Husserl aborde la distinction des expressions indépendantes («cheval», «maison») et des expressions dépendantes ("donc», «mais», « et») qui donne un sens légèrement différent à l'opposition de la signification normale et de la signification anormale. Les expressions dépendantes (« et»,

30. RL II/1, p. 83, [p. 98].

31. RL II/1, p. 83-84, [p. 98]. 
«ne... pas », «ou », etc.) sont des expressions qui possèdent une signification dite "dépendante ». En clair, à la différence d'expressions telles que «cheval », " table » ou « ciel »- qui sont des expressions qui se comprennent d'elles-mêmes -, les expressions dependantes ou syncatégorématiques ne peuvent avoir un sens que lorsqu'elles sont amalgamées à d'autres expressions. Au sein de l'énoncé "Les couteaux et les fourchettes sont dans le tiroir», le mot «et»a très certainement une signification. Et cette signification est déterminée par le contexte de la proposition qui fait en sorte que le mot " et » etablit un rapport de conjonction entre les couteaux et les fourchettes. Comme le note avec justesse Jocelyn Benoist, à partir du moment où l'on s'installe au sein du régime de la signification, il n'y a aucun élément du discours qui ne soit pas une signification ${ }^{32}$.

Est-ce à dire que le mot « et » n'a aucune signification lorsqu'il est pris isolément? Il s'agit sans aucun doute de la conclusion que l'on retrouve chez Wittgenstein : en dehors de ses différents usages, un mot n'a pas de signification ${ }^{33}$. La position de Husserl relativement à cette question est plus contrastée. En dépit du fait que le mot «et » n'a pas la signification qu'il peut avoir dans le contexte d'une proposition, il a tout de même une signification. Le pari de Husserl est donc de reconnaître que l'usage au sein d'une proposition détermine normalement le sens des expressions syncatégorématiques tout en refusant d'éponger la signification dans l'usage. Ce tour de force conduit Husserl à faire une distinction intéressante entre la signification normale et la signification anormale d'une expression. Dans le contexte de la proposition, « et » possède une signification claire et précise, il exprime sa signification normale. Par contre, lorsque je le considère d'une maniere isolée en dehors de la place qu'il occupe habituellement dans le contexte d'une proposition, le mot « et » possède toujours une signification quoique cette dernière soit dite anormale 34 .

Bien sûr, lorsque le mot «et» est rencontré en dehors de tout usage propositionnel, il ne remplit alors aucune fonction syntaxique. Il ne peut donc pas avoir sa signification normale. Toutefois, ce n'est pas parce que le mot « et » ne relie rien qu'il ne possède aucun rôle grammatical. Pour s'en convaincre, il suffit de songer à l'exemple suivant: " "Et" est un mot très utile ". Dans cette proposition, le mot " et » possède bel et bien une signification même s'il ne remplit pas sa fonction grammaticale habituelle. Le mot « et » joue le rôle non pas de connecteur syntaxique mais de sujet grammatical. Une telle modification de la fonction grammaticale du terme « et s'explique par le fait que le mot «et» a été nominalisé. Par le truchement des

\footnotetext{
32. J. Benoist, Intentionnalité et langage dans les Recherches logiques de Husserl, Paris, Presses universitaires de France, 2001, p. 94.

33. L. Wittgenstein, Le cahier bleu et le Cahier brun, tr. fr. G. Durand, Paris, Gallimard, 1965, p. 164.

34. RL II/1, p. 316, [2e tome, $2^{\mathrm{e}}$ partie, p. 110].
} 
guillemets, on a transformé une expression syncatégorématique en expression catégorématique. Dans l'exemple précédent, « et » ne remplit plus le rôle d'une conjonction, mais il est un nom qui nous permet de désigner l'opération de la conjonction. L'expression devient pour ainsi dire son propre nom. Or, c'est justement en vertu de cette modification toujours possible de la signification des expressions syncatégorématiques que le mot «et» pris isolément n'est pas une coquille vide de signification. Étant donné que le mot «et»est maintenant considéré comme une expression autonome et complète comme n'importe quelle expression catégorématique, on n'a pas besoin de l'insérer dans le contexte d'une proposition pour lui donner un sens plein et entier. L'expression « et » possède donc une signification anormale qui fait en sorte que je peux, au moins d'une manière minimale, considérer ce signe comme étant doté d'une signification.

Dans les Recherches logiques, Husserl nomme ce phénomène $\mathrm{l}^{\prime}$ hétérogrammaticalité ${ }^{35}$. Il est en principe toujours possible d'arracher une expression à ses coordonnées grammaticales habituelles pour lui donner un autre sens. Cette modification de la grammaire de certaines expressions qui permet de les faire basculer de leur signification normale à leur signification anormale n'a rien d'accidentel ou de contingent. En fait, cette possibilité est inscrite dans l'essence de tout langage et elle est justement ce qui nous permet de tenir un discours sur la signification ${ }^{36}$. Sans en faire un inventaire exhaustif et détaillé, Husserl reconnaît l'existence de divers modificateurs de signification. Jusqu'à maintenant, nous avons rencontré la modification de signification par le truchement des guillemets. Lorsque je dis que la Terre est ronde, mon discours porte sur le fait, scientifiquement établi, que la Terre est ronde. Par contre, lorsque j'emploie cette proposition entre guillemets, je fais un usage différent et anormal de la proposition qui vous pousse à porter davantage attention à la proposition et à la signification qu'elle exprime. La métaphore est un autre exemple d'usage « anormal » du langage. Et, finalement, il y a toute une kyrielle de prédicats (vrai, fictif, etc.) qui ont l'aptitude de transformer la signification de certains termes. Pour reprendre l'exemple de Husserl, lorsque je dis que le centaure est une fiction de poète, le mot «fiction » vient modifier le sens de « centaure $»^{37}$.

Mon intention n'est pas ici d'appliquer aveuglément la théorie des expressions syncatégorématiques aux expressions indexicales. Les expressions indexicales possèdent une autonomie sémantique beau-

\footnotetext{
35. RL II/1, p. 323, [p. 116].

36. « Il y a dans le domaine de la signification des lois a priori selon lesquelles des significations, tout en conservant un noyau essentiel, doivent se transformer de diverses manières en de nouvelles significations ». (RL II/1, p. 324, [p. 116]).

37. RL II/2, p. 323, [p. 118].
} 
coup plus forte qui permet à certaines d'entrent elles de fonctionner comme des pronoms ( «je », «tu », «il », etc.) et d'accomplir la fonction grammaticale des expressions catégorématiques. De surcroît, à la différence des expressions syncatégorématiques, on peut imaginer des cas où des expressions comme "Maintenant! ", " Ici ! » ont une signification autonome tout à fait compréhensible. En dépit de l'importance de ces observations, il n'en demeure pas moins que les indexicaux possèdent certaines particularités grammaticales qui font en sorte que l'on ne peut pas les traiter comme des noms. Une fois cette précaution prise, je crois qu'il est tout à fait légitime d'imaginer un nouvel usage des expressions indexicales qui consisterait à les nominaliser en utilisant les guillemets. Bien sûr, cette manœuvre produit un effet différent que dans le cas des expressions syncatégorématiques, mais le fait demeure que nous sommes en présence d'un phénomène d'hétérogrammaticalité qui met à l'avant-plan la signification générale et anormale des indexicaux ${ }^{38}$.

Le $\S 53$ des Méditations cartésiennes ne fait d'ailleurs que confirmer cette hypothèse. A première vue, cette section ne semble pas être pertinente dans le cadre de cet article puisque les expressions indexicales ne sont pas guillemetées, mais mises en italiques. À bon droit, Elisabeth Ströker conteste ce choix des éditeurs des Husserliana puisque le texte manuscrit de Husserl emploie les guillemets et non pas les italiques ${ }^{39}$. Une fois cette précision faite, la lettre du texte de Husserl concorde parfaitement avec mon interprétation des guillemets phénoménologiques. Dans ce passage capital de la cinquième Méditation explicitant la structure fondamentalement égocentrique du monde primordial qui s'organise autour de l'opposition de $\mathrm{l}^{\prime}$ « Ici » et du «Là-bas », Husserl fait un usage des guillemets et des indexicaux correspondant tout à fait à celui que je viens d'évoquer. Les premières lignes du $\S 53$ suffiront à illustrer ce point :

Mon corps propre physique a, en tant que référé à lui-même, le mode de donnée de l'"Ici " central; tout autre corps, et par conséquent celui de l'«Autre », a le mode du "Là-bas ». Cette orientation du là-bas est, grâce à mes kinesthèses, sujet du libre changement ${ }^{40}$.

Cet extrait est important pour au moins trois raisons. 1/ D'une part, ce texte confirme le rapprochement proposé un peu plus haut

\footnotetext{
38. Barry Smith et Kevin Mullingan proposent aussi un tel rapprochement. B. C. Smith et K. Mulligan, "A Husserlian Theory of Indexicality », Gräzer philosophische Studien, vol. 29, p. 133-163.

${ }^{39}$. E. Ströker, "Editorischer Bericht», in Cartesianische Meditationen, 2e édition, Hamburg, Felix Meiner Verlag, 1987, p. XXXII. En dépit de l'importance de la correction, il ne faudrait pas sombrer dans un fétichisme de la typographie. Que l'on utilise les italiques ou les guillemets, l'important est de noter l'utilisation d'une stratégie typographique pour marquer l'altération de la signification normale.

40. CM, p. 145-146, [p. 165].
} 
entre les indexicaux et les expressions syncatégorématiques. Afin de mettre en relief la nominalisation présupposée par l'usage des guillemets, Husserl traite grammaticalement les indexicaux encadrés de guillemets comme des noms (c'est-à-dire des expressions catégorématiques) en les écrivant avec une majuscule. 2/ Deuxièmement, on voit très bien dans ce passage que seule la signification universelle du déictique «Ici » est retenue par Husserl. En tant qu'individu empirique, je peux occuper différents lieux de l'espace. Par contre, mon corps propre est toujours "ici » puisque ce dernier sert de point de réference à l'opposition de $l^{\prime}$ «ici» et du «là-bas ». 3/ Finalement, Husserl fait une distinction entre le «Là-bas » qui est une structure de l'orientation de mon corps propre et le là-bas (sans les guillemets) qui renvoie, selon les contextes, à différents lieux de l'espace.

\section{Conclusion}

Selon un reproche plutôt fréquent que l'on adresse à la phénoménologie, cette dernière serait coupable de tenter de court-circuiter le langage en entreprenant de décrire des structures aprioriques qui se situent en amont des significations linguistiques. Ce geste paraît être absurde et paradoxal puisque le discours phénoménologique doit tout de même donner une forme langagière à ses descriptions et ses analyses. La conclusion vers laquelle nous acheminent nos analyses est que la méthode phénoménologique ne consiste pas à enjamber la grammaire de notre langage pour décrire l'expérience dans son immédiateté brute. Comme le montre l'usage méthodologique que Husserl fait des guillemets et de la nominalisation dans les Méditations cartésiennes et ailleurs, le discours phénoménologique exploite certains dispositifs de la grammaire de notre langage pour se mettre en place.

La présente reconstruction de la théorie husserlienne des guillemets phénoménologiques permet d'écarter une autre objection qui, d'un point de vue philosophique, s'avère beaucoup plus sérieuse. Plusieurs lecteurs de Husserl ont remarqué le lien étroit qu'il y a entre le discours indirect (oratio obliqua) et le discours phénoménologique ${ }^{41}$. Comme j'ai déjà eu l'occasion de le mentionner dans mon propos liminaire, David Smith et Ronald McIntyre souscrivent à une thèse de ce type. Le problème évident que pose une telle approche est qu'elle rend la phénoménologie totalement incapable de rendre compte de la transcendance de la réalité extérieure. En effet,

\footnotetext{
${ }^{41}$. Pour une reconstruction différente de la spécificité de l' «oratio » phénoménologique qui s'en tient à la période des Recherches logiques, cf. Pierre-Jean Renaudie, "Rectitude et obliquité intentionnelle de l'Oratio phénoménologique: Remarques croisées sur McDowell, Brentano et Husserl », Bulletin d'analyse phénoménologique, vol. 6, no 8, 2010, p. 108-128.
} 
lorsque j'emploie le discours indirect pour rapporter les paroles d'autrui, la question de la vérité des propos rapportés est mise entre parenthèses. Par conséquent, on voit difficilement comment on peut interpréter le discours phénoménologique sur le patron du discours indirect sans risquer de démagnétiser la transcendance du monde extérieur. Cette conviction mène $\mathrm{d}^{\prime}$ ailleurs Smith et McIntyre à proposer de «corriger » la conception husserlienne de l'intentionnalité en lui annexant une nouvelle théorie de la référence qui repose sur une analyse différente de la logique des expressions indexicales et démonstratives ${ }^{42}$.

Une telle entreprise de reconstruction possède un intérêt philosophique relativement indépendant de la lettre des textes de Husserl. Rejeter cette interprétation audacieuse sous prétexte qu'elle ne donne pas un portrait adéquat de la pensée de Husserl, ce serait sans doute rater l'essentiel. Par contre, si nous sommes soucieux de comprendre en quoi consiste véritablement la position de Husserl, je crois qu'il convient d'insister sur la particularité et l'originalité de l'usage phénoménologique des guillemets qui nous interdit de le rabattre simplement sur la figure du discours indirect. Notre parcours nous permet donc de préciser de quelle manière Husserl exploite les ressources que nous offre la grammaire de notre langage tout en revendiquant la spécificité du registre du discours phénoménologique.

42. D. Smith et R. McIntyre, p. 213. 